\title{
On the Detectability of Planet X with LSST
}

\author{
David E. Trilling $^{1,2}$ (D), Eric C. Bellm ${ }^{3}$ (D), and Renu Malhotra ${ }^{4}$ (D) \\ ${ }^{1}$ Department of Physics and Astronomy, Northern Arizona University, Flagstaff, AZ 86011, USA; david.trilling@nau.edu \\ ${ }^{2}$ Lowell Observatory, 1400 W Mars Hill Road Flagstaff, AZ 86001, USA \\ ${ }^{3}$ Department of Astronomy, University of Washington, Box 351580, Seattle, WA 98195, USA \\ ${ }^{4}$ Lunar and Planetary Laboratory, The University of Arizona, 1629 E University Boulevard, Tucson AZ 85721, USA \\ Received 2018 February 18; revised 2018 April 3; accepted 2018 April 18; published 2018 May 18
}

\begin{abstract}
Two planetary mass objects in the far outer solar system — collectively referred to here as Planet X- have recently been hypothesized to explain the orbital distribution of distant Kuiper Belt Objects. Neither planet is thought to be exceptionally faint, but the sky locations of these putative planets are poorly constrained. Therefore, a wide area survey is needed to detect these possible planets. The Large Synoptic Survey Telescope (LSST) will carry out an unbiased, large area (around $18000 \mathrm{deg}^{2}$ ), deep (limiting magnitude of individual frames of 24.5) survey (the "wide-fast-deep (WFD)" survey) of the southern sky beginning in 2022, and it will therefore be an important tool in searching for these hypothesized planets. Here, we explore the effectiveness of LSST as a search platform for these possible planets. Assuming the current baseline cadence (which includes the WFD survey plus additional coverage), we estimate that LSST will confidently detect or rule out the existence of Planet X in $61 \%$ of the entire sky. At orbital distances up to $\sim 75$ au, Planet X could simply be found in the normal nightly moving object processing; at larger distances, it will require custom data processing. We also discuss the implications of a nondetection of Planet X in LSST data.
\end{abstract}

Key words: Kuiper belt: general - methods: observational - parallaxes - planets and satellites: detection - surveys

\section{Introduction}

The possibility of undiscovered planets in the solar system has long fascinated astronomers and the public alike. Recent studies of the orbital properties of very distant Kuiper Belt objects (KBOs) have identified several anomalies that may be due to the gravitational influence of one or more undiscovered planetary mass objects orbiting the Sun at distances comparable to the distant KBOs. Trujillo \& Sheppard (2014) and Sheppard \& Trujillo (2016) noted a clustering of the argument of perihelion (the angular position of the perihelion relative to the ascending node of an orbit on the J2000 reference plane) of KBOs whose semimajor axes exceed 150 au. Subsequently, Batygin \& Brown (2016) and Brown \& Batygin (2016) noted a clustering of the longitudes of perihelion and of the orbit poles of the same group of distant KBOs. Malhotra et al. (2016) noted that the most distant KBOs have near-integer period ratios, suggestive of dynamical resonances with a massive perturber.

These orbital distribution peculiarities could be caused by an unseen massive body. Trujillo \& Sheppard (2014) estimate a super-Earth mass object orbiting at a heliocentric distance $\gtrsim 250$ au (while noting that a range of parameters for an unseen parameter could produce the observational signature that they observe); Brown \& Batygin (2016) estimate a planet of mass 5-20 $M_{\oplus}$ in an orbit of semimajor axis 380-980 au, perihelion distance $150-350$ au and moderately inclined $\left(\sim 30^{\circ}\right)$ to the ecliptic; and Malhotra et al. (2016) suggest a $\sim 10 M_{\oplus}$ planet in an orbit of semimajor axis $\sim 665$ au of moderate eccentricity and two possible inclinations $\left(i \approx 18^{\circ}\right.$ or $i \approx 48^{\circ}$ to the ecliptic). The observational sample size for the above analyses is relatively small, 6-13 objects, depending upon choice of perihelion distance cut-off. Finally, in a separate line of argument, Bailey et al. (2016) and Gomes et al. (2017) show that the obliquity of the Sun of around $6^{\circ}$ can be explained by a 10-20 $M_{\oplus}$ perturber with a semimajor axis of 400-600 au.
Separately, for a larger sample of $\sim 160$ distant KBOs whose semimajor axes are in the range 50-80 au, Volk \& Malhotra (2017) reported a strong deviation of the mid-plane from the solar system's invariable plane. Based on this deviation, they suggest the presence of a smaller planetary mass object of mass $0.1-2.4 M_{\oplus}$ at distance $60-100 \mathrm{au}$ in an orbit inclined to the ecliptic by a few to a few tens of degrees.

The predicted locations (in the sky) and brightnesses for these massive unseen objects-here referred to collectively as "Planet X"- are sufficiently unconstrained that large area sky surveys must be carried out. Such surveys require moderately large telescopes - a distant planet may be as bright as $V=15$ (Volk \& Malhotra 2017) or as faint as $V=22-25$ (Brown \& Batygin 2016) — and very large fields of view, as the search regions are at least hundreds of square degrees, and could easily be thousands.

The Large Synoptic Survey Telescope (LSST), when it comes online in 2022 , will easily meet these criteria, with its single exposure depth of $r \sim 24.5$ and field of view of $9.6 \mathrm{deg}^{2}$. Furthermore, and more powerfully, some $85 \%$ of the 10-year LSST program will be used for the "wide-fast-deep" (WFD) survey in which a large portion of the available sky (roughly $-60^{\circ}-0^{\circ}$ decl.) will be observed around 100 times in each of six filters (ugrizy), over 10 years. (We note that the galactic plane is not included in the WFD coverage.) The total sky coverage of this WFD survey will be around $18000 \mathrm{deg}^{2}$, or around $44 \%$ of the entire sky. In other words, LSST will be an excellent facility to search for Planet X in the southern sky.

Here, we explore the possibility that LSST can be used to detect Planet X, if it exists.

\section{Detecting Planet X with LSST}

In order for LSST to detect Planet $X$ several simple requirements must be met, as follows. (1) The planet must be within the nominal LSST sky coverage in the current baseline observing cadence (minion_1016) as simulated by the LSST 


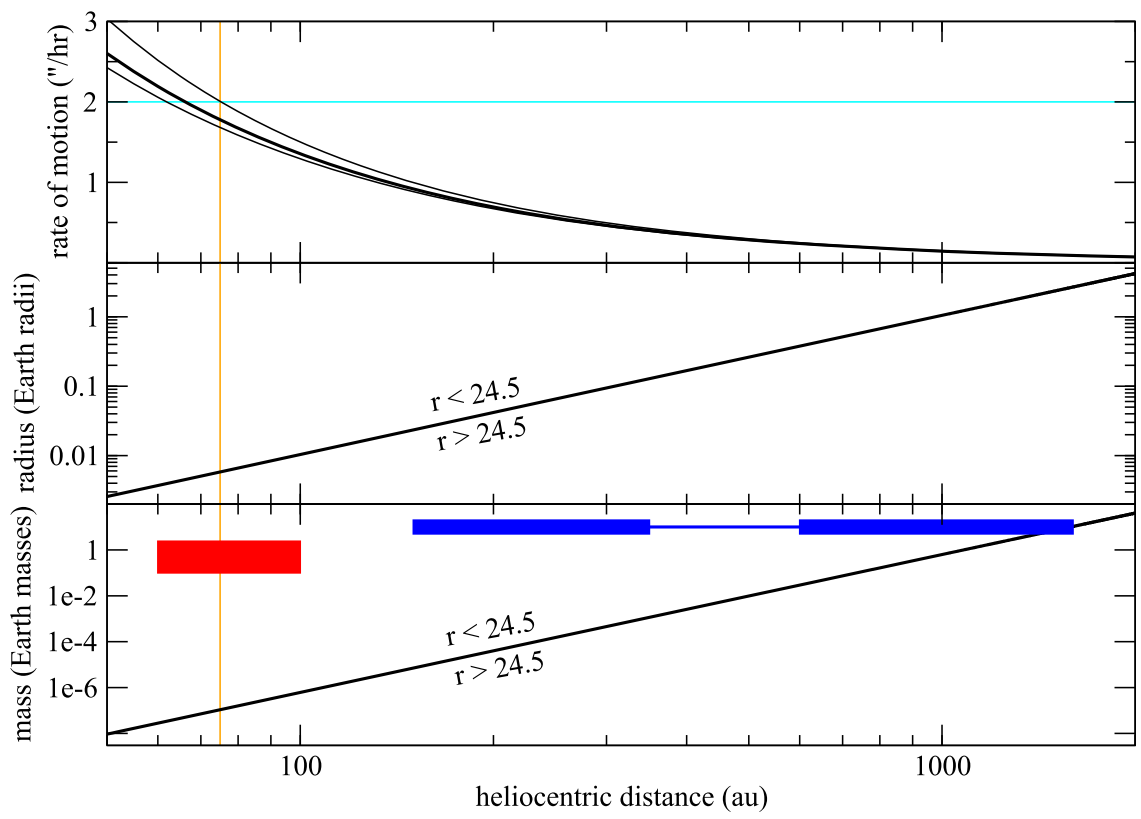

Figure 1. Top panel: sky rate of motion as a function of heliocentric distance. The thick central line shows the rate for an object on a circular orbit observed at opposition. The thin black lines show the rates of motion for orbits with eccentricity near unity for objects near pericenter (lower curve) and apocenter (upper curve). For a perturber located at a particular heliocentric distance, the on-sky rate of motion for eccentric orbits lies either below or above the rate for circular orbits; the upper and lower curves show the range of on-sky rate of motion for the entire possible range of eccentricities, zero to unity. The light blue line at 2 arcsec hr $^{-1}$ shows the detectability limit for objects to be detected in nightly processing; only objects closer than 75 au (orange vertical line in all panels) would be detected in this standard processing. Middle and bottom panels: radius (in Earth radii) and mass (in Earth masses) of objects at the LSST detection limit of $r=24.5$. Detectable objects are above these lines. In the bottom panel, the Volk-Malhotra object is shown as the red rectangle and the range of perihelion and aphelion locations for the TrujilloSheppard/Batygin-Brown object is shown as the blue rectangles; the thin blue line connects these regions and shows the entire range of possible locations.

Operations Simulator (Connolly et al. 2014; Delgado \& Reuter 2016; Reuter et al. 2016). (2) The planet must be bright enough to be detected by LSST. In a single exposure this implies $r \leqslant 24.5$, but through stacking images, deeper searches are possible. (3) The planet must have an on-sky rate of motion that is detectable with LSST frames. The timescale on which a distant planet can be detected depends on its rate of motion and therefore its distance, and different rates require different data analysis techniques. (4) The LSST cadence must be commensurate with detecting the planet's motion.

In the sections that follow, we assume that the ability to detect point sources within LSST's sky coverage is perfect. In other words, we assume that every point source above the $5 \sigma$ limit of $r \sim 24.5$ is detected perfectly. We ignore the effects of crowded fields.

\subsection{Coverage and Cadence}

The LSST field of view is approximately circular with a diameter of 3.5 deg (Ivezic et al. 2008). A distant solar system object at $\{60,1000\}$ au has an apparent motion on the sky in the range of $\{2.5,0.15\} \mathrm{arcsec}^{-1}$ or $\{6.1,0.37\} \mathrm{deg} \mathrm{yr}^{-1}$ (Figure 1). This rate of motion is dominated by the reflex motion of the Earth and is close to the parallactic motion; the orbital motion of a distant body contributes only a small fraction to its on-sky rate of motion.

Therefore, an object at 100 au will most likely stay within one or two LSST pointings per observing season, and an object at 1000 au will most likely stay within one or two LSST pointings over the 10-year LSST baseline survey. Here, we consider the case of searching for distant moving objects within a single observing season (the months in a year when a given field is above two airmasses at night and therefore could be observed by LSST), which means across multiple visits to a single LSST pointing. ${ }^{5}$ (A pointing is a single fixed piece of sky that LSST returns to over and over again.)

For a pointing to be successfully searched, we require five (or more) visits within 45 days-a nominal value that approximates when a field is best placed for observationsand that at least two pairs of observations are separated by two (or more) days each. The former requirement ( 45 days, five visits) ensures that a reasonably long observational arc is obtained; the latter (two-day separation) ensures that object motion can be detected (though this turns out to be a conservative assumption, as shown below). We note that the nominal observing requirement for the LSST WFD survey is that any field that is observed once in a night must be observed again $\sim 30$ minutes later in that same night. Thus, there are enough measurements across a season ( 45 days) to identify and measure the motion of a distant object; five (or more) detections is a conservative requirement that yields confidence in a consistent set of detections (minimizing the risk that other astrophysical sources or noise have contaminated the linked detections). Requiring at least two visits to be separated by more than two days allows us the possibility of finding distant objects, as the intra-night motion of objects at 1000 au is less than one arcsecond and may not be easily detected (as described below).

Figure 2 shows the result of querying the current LSST baseline cadence (minion_1016) against these requirements. Tan colored areas show LSST pointings that satisfy the above requirements, and purple show areas that do not. (Gray regions

\footnotetext{
5 To simplify the exposition, we describe searching for Planet $\mathrm{X}$ within a single LSST pointing. However, LSST moving object searches are undertaken without respect for field boundaries. As adjacent fields have very similar observation cadences, Planet $\mathrm{X}$ would still be identified if it moved between two pointings in a single observing season.
} 


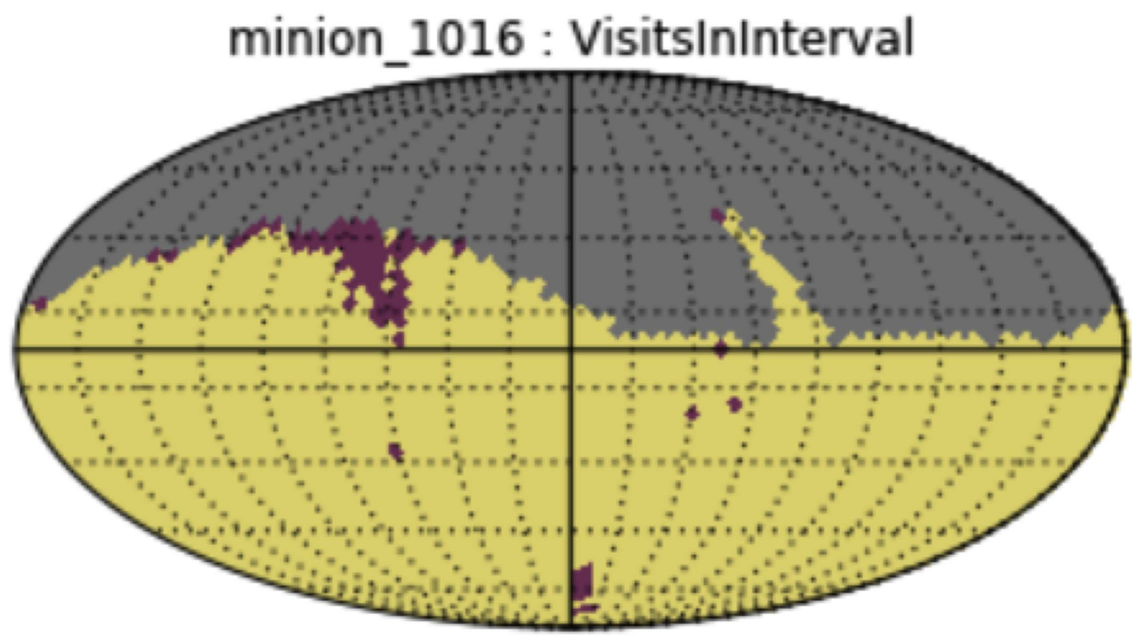

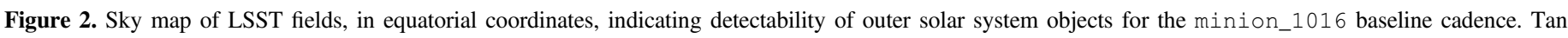

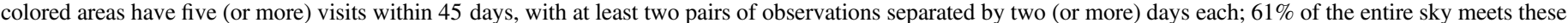

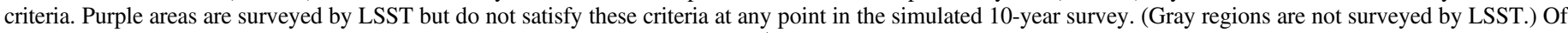

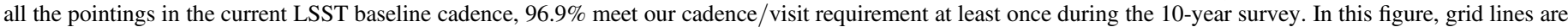
marked every $20^{\circ}$, and the plot center is R.A. of $180^{\circ}$, with R.A. increasing to the right from $0^{\circ}$ to $360^{\circ}$.

are not surveyed by LSST.) We note that minion_1016 includes the WFD sky coverage as well as the North Ecliptic Spur, the South Celestial Cap, and the Galactic Plane. Some $44 \%$ of the entire sky is surveyed in the baseline WFD survey, and $63 \%$ of the sky is covered by some portion of the LSST survey. Of all the pointings in the current LSST baseline survey, $96.9 \%$ meet our requirements at least once during the 10 -year survey. This corresponds to $61 \%$ of the entire sky.

\subsection{Detections and Data Processing}

Almost all moving objects detected in LSST data will be found through image differencing. Standard LSST image differencing subtracts a pre-existing coadded template image from each new visit image. The current Moving Object Pipeline Software (MOPS) design (e.g., Jones et al. 2016; Vereš \& Chesley 2017) first generates "tracklets" by connecting difference image detections obtained in multiple visits within a night. The median intra-night gap for visit pairs in the current LSST baseline cadence (minion_1016 at the time of this writing) is about 30 minutes. Whether MOPS will recognize slow-moving outer solar system objects will depend on their rate of motion, the gap between visits within a night, and the details of the LSST source association and MOPS pipelines. Therefore, given the expected ${ }^{6}$ LSST PSF of around 0.8 arcsec, moving point sources with separations of $\gtrsim 1 \operatorname{arcsec}$ between the two images should be detected and linked through standard LSST nightly processing. This corresponds to objects having a sky motion $\gtrsim 2 \operatorname{arcsec~hr}^{-1}$, equivalent to orbital distance $\lesssim 75$ au (Figure 1). Therefore, given standard LSST nightly processing, the parameter space of Planet X-like objects would be partially detectable for the Volk-Malhotra object but not at all detectable for the Trujillo-Sheppard/Batygin-Brown objects.

Possible planets that are beyond 75 au will move too slowly to be detected in standard LSST processing - in this case, two detections within a night would be associated into a single nonmoving object—so additional strategies must be employed

\footnotetext{
6 http://opsim.lsst.org/runs/reference_run/minion_1016/summary_ minion_1016.pdf
}

to search for those objects. If images from adjacent nights are compared, objects with motions $\gtrsim 0.04 \mathrm{arcsec} \mathrm{hr}^{-1}$ would be detectable (assuming a $24 \mathrm{hr}$ baseline). The corresponding distance for this motion is $<2000 \mathrm{au}$; all possible orbital parameters for Planet $\mathrm{X}$ produce faster motions than this (Figure 1). Here, the process is the same as the nightly (standard processing), but such night-to-night comparisons and linking will not be provided by the LSST project. (Night-tonight comparisons from nonadjacent nights can be treated identically to those for adjacent nights.)

Direct imaging provides an alternative approach to identifying distant moving objects. Yearly LSST Data Release Processing will provide parallax and proper motion estimates for objects in noncrowded fields; at $r \sim 24$, these have accuracies of 2.9 mas and 1.0 mas year $^{-1}$, respectively, over 10 years (LSST Science Collaboration et al. 2009) - easily sufficient to detect the motion of all proposed Planet X-like objects.

\section{Results and Discussion}

In Figure 1, the middle and lower panels show the radius (assuming albedo of 0.5) and mass (assuming density of $5.5 \mathrm{~g} \mathrm{~cm}^{-3}$, same as the Earth's density) of objects at the photometry detection threshold of LSST $(r=24.5)$. Detectable objects $(r<24.5)$ are above the lines. Brown \& Batygin (2016)s proposed distant object solution-5-20 Earth mass object with perihelion of $150-350$ au and aphelion of 600-1600 au-would be easily detectable at perihelion, and detectable at aphelion except for a small slice of parameter space at the largest possible distance and smallest allowed mass (a region that becomes marginally more searchable through stacking intra-night images). In other words, for the $96.9 \%$ of the LSST sky coverage where the cadence will allow detection, the Trujillo-Sheppard/Batygin-Brown object is very likely to be detected. Either night-to-night comparisons or direct imaging would be necessary to detect this slow-moving object. The Volk-Malhotra object is detected far more easily, as it is much brighter (i.e., more massive) than the detection limit shown in Figure 1. In some cases, standard MOPS processing might reveal the Volk-Malhotra object; in other cases, night-tonight comparisons or direct imaging may be required. 
In general, LSST should be able to detect both the TrujilloSheppard/Batygin-Brown object and the Volk-Malhotra object. The Volk-Malhotra object would be closer and therefore faster moving. (It is also predicted to be brighter than the Trujillo-Sheppard/Batygin-Brown object.) In general, greater sky rate of motion makes detection and linking with MOPS easier. Objects moving faster than about $0.7 \operatorname{arcsec~hr}^{-1}$ (i.e., closer than about $200 \mathrm{au}$ ) should be readily detectable in night-to-night processing, having moved some 17 arcsec in $24 \mathrm{hr}$. Such an object will move only around 13 arcmin during the 45 day window specified above, and so would be likely to stay within a single LSST pointing during the entire observing season.

In these calculations, we have used the current LSST baseline cadence (minion_1016). The LSST project is also actively considering a rolling cadence that increases the number of visits to a given field in an observing season. If anything, the rolling cadence would increase the detectability of Planet X, giving more detections in a single season (as we have shown above that the most likely detection scenario is with intra- or inter-night detections, not year-to-year).

Brown \& Batygin (2016) aggregated a large number of previous and ongoing surveys to show regions of the sky where their proposed object can presently be ruled out and identified regions where it could still exist undetected. They find that the most likely region on the sky for Planet $X$ is R.A. $\approx 2-10 \mathrm{hr}$ and decl. of around $-20^{\circ}$ to $+10^{\circ}$, where the object would be nearer its aphelion and therefore slowly moving (roughly $0.1 \operatorname{arcsec~hr}^{-1}$ ) and faint ( $V$ of 22-25). This rate of motion would require night-to-night comparisons, but this is not infeasible; the apparent magnitude also is mostly brighter than the LSST detection limit (Figure 1). Interestingly, however, this region of sky is the part of the baseline LSST cadence that is most likely to fail to meet our cadence requirements (Figure 2).

LSST is likely to spend significant time surveying the socalled North Ecliptic Spur: the part of the ecliptic plane that is north of the nominal WFD survey coverage. (Coverage of the North Ecliptic Spur is shown in Figure 2 as the region north of the celestial equator.) The cadence for surveying this region of the sky may be optimized for nearby solar system objects and is not likely to significantly affect the discovery space for distant solar system objects. It is reasonable to assume that the detection efficiency in the North Ecliptic Spur is as good, or better, than in the nominal WFD survey area. Our probability of detecting Planet X with LSST is thus most affected by the total sky area covered rather than the details of the cadence.

The high stellar density in the galactic plane will make detections of distant moving objects there more difficult. If Planet X exists there, it is less likely to be detected than if it exists in less-crowded regions of the sky. In the case of the nondetections of Planet $\mathrm{X}$, the reduced detection efficiency there naturally weakens any conclusions to be drawn from that region of sky, but those data will still be useful to place constraints once the detection efficiency is measured.

A nondetection with LSST would yield a map of regions of the sky where planets brighter than $r=24.5$ with a corresponding map of size and distance are ruled out; this map would only apply to the $63 \%$ of the sky that will be surveyed by LSST. From such a map, we could compute the probability of ruling out the existence of a Mars-to-Earth size object at 60-100 au and, similarly, for a Neptune-size object at $150-1600$ au. If these probabilities are close to unity, the recently proposed hypotheses for distant planets would lose support. It would then be necessary to re-evaluate alternative explanations for the anomalies in the orbital properties of distant KBOs. Two alternatives are that the anomalies are statistical flukes, or that the anomalies indicate a relatively recent perturbation to the distant solar system such as by a close stellar flyby. The "statistical fluke" explanation is also testable by LSST because of the large number of distant KBOs that LSST will discover, which will allow much better statistical analysis of their orbital properties and re-assessment of the evidence for Planet X.

\section{Conclusion}

If the putative Planet $\mathrm{X}$-either the Trujillo-Sheppard/ Batygin-Brown object or the Volk-Malhotra object-exists, is within the area of sky that LSST will cover, and is brighter than the LSST single exposure depth, then there is a very high probability that it will be detected, either with standard processing or through custom processing. For orbital distances closer than about $75 \mathrm{au}$, the rate of motion is fast enough that it can be detected in the standard LSST moving object nightly processing. More involved data processing is required to detect objects that are more distant. Should there be no detection of distant planet(s) in LSST data, the orbits (and potential anomalies thereof) of the many distant Kuiper Belt Objects that LSST will discover will help to constrain the properties and existence of Planet $\mathrm{X}$ in regions of the sky not surveyed (or inadequately surveyed) by LSST.

As we were completing this work, we became aware of a third, independent line of argument by Silsbee \& Tremaine (2018) who suggest that an unseen Mars-to-Earth sized body in an orbit inclined about 30 degrees to the ecliptic and of perihelion distance 40-70 au and semi-major axis less than 200 au could account for the properties of the detached KBOs (a population of KBOs with perihelion distance exceeding $\sim 38$ au and semi-major axes 80-500 au). This suggested object is similar in mass to the one suggested by Volk \& Malhotra (2017), spans a similar range of ecliptic latitude, but a larger range of heliocentric distance (40-360 au). Our conclusions for the putative Planet $\mathrm{X}$ described above apply to this object as well.

D.E.T. acknowledges support from the Office of the Vice President for Research at Northern Arizona University. R.M. is grateful for research support from NASA (grant NNX14AG93G) and NSF (grant AST-1312498). An anonoymous referee provided a number of suggestions that improved this manuscript.

Software: LSST metrics analysis framework (Jones et al. 2014).

\section{ORCID iDs}

David E. Trilling (i) https://orcid.org/0000-0003-4580-3790

Eric C. Bellm (1) https://orcid.org/0000-0001-8018-5348

Renu Malhotra (ㄴ) https://orcid.org/0000-0002-1226-3305

\section{References}

Bailey, E., Batygin, K., \& Brown, M. E. 2016, AJ, 152, 126

Batygin, K., \& Brown, M. E. 2016, AJ, 151, 22

Brown, M. E., \& Batygin, K. 2016, ApJL, 824, 23

Connolly, A. J., Angeli, G. Z., Chandrasekharan, S., et al. 2014, Proc. SPIE, 9150, 915014

Delgado, F., \& Reuter, M. A. 2016, Proc. SPIE, 9910, 991013 
Gomes, R., Deienno, R., \& Morbidelli, A. 2017, AJ, 153, 27

Ivezic, Z., Tyson, J. A., Abel, B., et al. 2008, arXiv:0805.2366

Jones, R. L., Yoachim, P., Chandrasekharan, S., et al. 2014, Proc. SPIE, 9149 , 91490B

Jones, R. L., Jurić, M., \& Ivezić, Ž. 2016, in IAU Symp. 318, Asteroids: New Observations, New Models (Cambridge: Cambridge Univ. Press), 282

LSST Science Collaboration, Abell, P. A., \& Allison, J. 2009, arXiv:0912.0201
Malhotra, R., Volk, K., \& Wang, X. 2016, ApJL, 824, 22

Reuter, M. A., Cook, K. H., Delgado, F., et al. 2016, Proc. SPIE, 9911, 991125

Sheppard, S. S., \& Trujillo, C. A. 2016, AJ, 152, 221

Silsbee, K., \& Tremaine, S. 2018, AJ, 155, 75

Trujillo, C. A., \& Sheppard, S. S. 2014, Natur, 507, 471

Vereš, P., \& Chesley, S. R. 2017, AJ, 154, 13

Volk, K., \& Malhotra, R. 2017, AJ, 154, 62, (erratum, AJ 2017, 154, 212) 